\title{
DISTRIBUTION OF YOUNG STARS AND GAS IN THE SMALL MAGELLANIC CLOUD
}

\author{
J. LEQUEUX \\ Observatoire de Meudon \\ 92195 Meudon CEDEX, \\ France \\ and \\ Ecole Normale Supérieure
}

\begin{abstract}
The relations between the distributions of interstellar gas and young stars in the central parts of the SMC are discussed, based on their distributions over the sky, radial velocities and distances. There are large differences between stars and gas. The existence of two main complexes is confirmed. There is no strong evidence for a distance in depth between these complexes larger than about $10 \mathrm{kpc}$, although Cepheid observations suggest a large total depth in some regions. Further, more accurate observations of Cepheids, especially of their mean radial velocities, are required for a better understanding of the structure of the SMC.
\end{abstract}

\section{Introduction}

The structure of the Small Magellanic Cloud (SMC) has been the subject of much work and debate in recent years; in particular, long-standing problems are the extension in depth of the SMC, and the nature and extent of its fragmentation. A review by Mathewson and Ford (1984) centred on the distribution of neutral hydrogen, and the most recent review is in Westerlund (1990).

A cursory examination of SMC data shows i) that there are gas (HI) and young stars (and also young clusters and HII regions) all over the face of the SMC, including the wing and its extension towards the Large Magellanic Cloud (LMC); ii) that the correlation between the surface density of gas and of young objects is not very good; and iii) that the older objects (i.e. planetary nebulae and carbon stars) as well as the integrated light have a different, more regular distribution and different kinematics compared to gas and young objects; this will be discussed in other talks at this Symposium. Having said this, we still have no answer to the questions previously raised. To say more, we have to combine two-dimensional distribution of relevant objects over the sky with information on a third dimension provided either by distance measurements or, more indirectly, by radial velocity observations. This information on the gas and young objects is discussed here. Section 2 will discuss the evidence for an extent in depth of the SMC, and Section 3 the threedimensional relations between gas and young objects, with some emphasis on Cepheids.

\section{Evidence for an extent in depth of the SMC}

The existence of great depth in the SMC along the line of sight was first suspected by Johnson in 1961. Through stellar photometry and spectral classification of supergiants, and independently through studies of distances of Cepheids obtained from the period-luminosity (P-L) or periodluminosity-colour (P-L-C) relations, a number of authors have suggested large total depths from 
20 to $40 \mathrm{kpc}$. For a complete discussion and list of references see Martin et al. (1989 - MML), Westerlund (1990) and Feast (1990). However, these studies all suffer from the existence of a scatter in the absolute magnitudes of stars of any spectral class (or for Cepheids, of a given period) which is of the order of, or larger than, the effect to be measured. This has been clearly pointed out by Tully and Wolff (1984) for blue supergiants and by Welch et al. (1987 - WMMM) for Cepheids. A critical discussion of these intrinsic dispersions is a prerequisite to any final conclusion. I am not convinced by the large depths derived from supergiant studies.

The Cepheid data look more convincing. All authors agree that the period-luminosity relation for SMC Cepheids has a fairly large scatter. For example, WMMM find that while the dispersion around the mean infrared ( $\mathrm{J}, \mathrm{H}$ and $\mathrm{K}$ ) period-luminosity relation is about $0.13 \mathrm{mag}$ for LMC Cepheids, it is almost two times larger for SMC Cepheids. This raises several problems: i) what is the nature of the dispersion for the LMC? A part of it must be intrinsic, due to the finite width of the Cepheid instability strip. WMMM consider that it is almost entirely intrinsic. ii) If the dispersion for LMC Cepheids is intrinsic, is the intrinsic dispersion for SMC Cepheids the same? WMMM's answer is 'no'; it should be larger by $30 \%$ because of the difference in metallicities and of the larger metallicity spread in the SMC Cepheids. If all this is true, an additional dispersion of only 0.18 mag is needed to account for the observed magnitude width of the SMC P-L relation, corresponding to $\pm 5 \mathrm{kpc}$ along the line of sight. Using the H, B-V P-L-C relation instead, on which they place more weight, WMMM find a smaller additional dispersion for SMC Cepheids of $0.12 \mathrm{mag}$, corresponding to $\pm 3 \mathrm{kpc}$ only. The larger depth found by Caldwell and Coulson (1986) appears less reliable as these authors use observations in the visible which are more sensitive to reddening and metallicity effects. Although Mathewson et al. (1986 \& 1988 - MFV) have made Iv $(1.05 \mu \mathrm{m})$ photometry, their results are also less reliable than those of WMMM because they have ignored the intrinsic width of the P-L-C relation and have only a small number of Iv measurements per Cepheid, hence a poor knowledge of the luminosity and an overestimate of the dispersion in the P-L-C relation. Indeed, the correlation between the individual distance moduli for Cepheids in common between WMMM and MFV is poor (Fig. 1). I am thus inclined to prefer the smaller but probably real SMC depth given by WMMM.

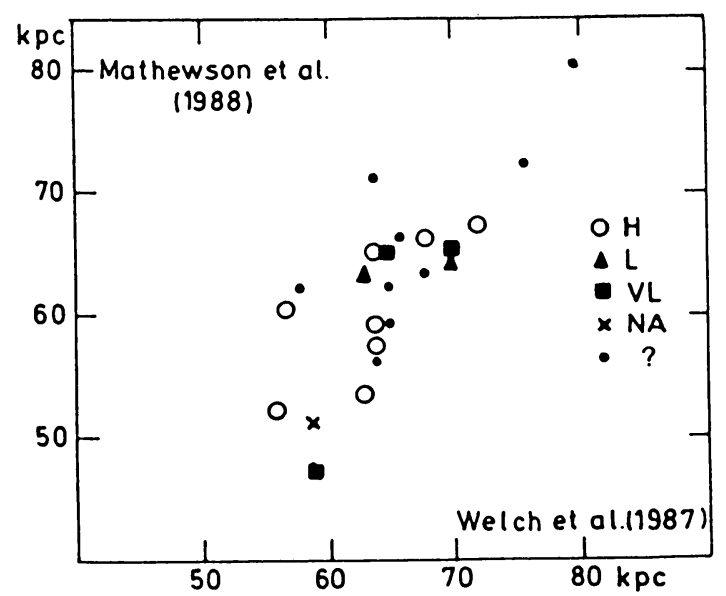

Figure 1. Comparison of distance moduli of individual SMC Cepheids obtained by Welch et al. (1987) and by Mathewson et al. (1988). Those Cepheids which can be assigned to one of the velocity groups of Martin et al. (1989) are represented by different symbols. Note the large scatter in the comparison and the absence of relation between velocity assignment and distance modulus. 
Another problem is that of a possible distance gradient throughout the SMC. It appears from the Cepheid work of Laney and Stobie (1986) and of WMMM that the wing is closer than the main body of the SMC. There also seems to be a gradient in distance from the NE to the SW of the bar. This is best seen in Figure 11 of MFV which shows that the Cepheids in their field 1, the active star-forming region at the SW of the Bar, are more distant on the average by about $7 \mathrm{kpc}$ with respect to the Cepheids in their fields 2 and 3, the centre and the NE of the bar, respectively.

\section{Relations between gas and young objects: toward a 3-D picture of the SMC}

Let us now consider the gas distribution as derived from the $21-\mathrm{cm}$ observations. The $21-\mathrm{cm}$ profiles as obtained by McGee and Newton (1981) are generally complex, showing two or more components at most positions. Mathewson and Ford (1984- MF) have built from these data a velocity-position diagram along a direction that they consider the major axis of the SMC (along the bar) and found two ridge-lines of $\mathrm{HI}$ intensity maxima: they define in this way two sub-systems, each one with a velocity gradient. Caldwell and Coulson (1986) connect the maxima in the same diagram in a different way and produce a very different picture, with one main component having a much larger velocity gradient. MMF have made a similar but deeper study using HI positionvelocity diagrams along 15 cuts distributed over the face of the SMC, and distinguish four different gas complexes. Component VL (for Very Low Velocity) has a mean radial velocity referred to the galactic centre V(GSR) between -50 and $-40 \mathrm{~km} / \mathrm{s}$, and is mainly concentrated in the SW part of the bar and its extension. Component $\mathrm{L}$, with $\mathrm{V}(\mathrm{GSR}) \approx-28 \mathrm{~km} / \mathrm{s}$ is a major feature visible over the centre and the SW part of the bar and extending to the $\mathrm{K} 1$ component in the wing. Component $\mathrm{H}$ (for High velocity) is another major component with $\mathrm{V}(\mathrm{GSR}) \approx 9 \mathrm{~km} / \mathrm{s}$ which covers essentially the whole extent of the SMC. Component VH is a weaker component at V(GSR) $\approx 30 \mathrm{~km} / \mathrm{s}$, detected only in the NE region of the bar and the beginning of the wing. It is probably connected with component $\mathrm{H}$. None of these components shows a strong velocity gradient. In fact the higher-velocity component of MF (that they call the mini-Magellanic Cloud) is the combination of $\mathrm{H}$ and $\mathrm{VH}$, and their lower-velocity component (their SMC remnant) is the combination of $\mathrm{L}$ and $\mathrm{VL}$. To ease comparisons between various authors, we note that $\mathrm{V}(\mathrm{GSR}) \approx \mathrm{V}$ (heliocentric) -160 $\mathrm{km} / \mathrm{s}$ and $\mathrm{V}(\mathrm{LSR}) \approx \mathrm{V}$ (heliocentric) $-11 \mathrm{~km} / \mathrm{s}$.

There are also CO observations in many regions of the SMC obtained either with the 1-m miniradiotelescope in Cerro-Tololo (Rubio et al.1990) or with the SEST (survey in progress by an ESO-Sweden collaboration). Molecular gas has been detected in the NE and SE of the bar and in the K1 region in the wing. Surprisingly, all the detected CO lines in the SW of the bar where the $\mathrm{H}$ and $\mathrm{L}$ components have comparable intensities in the HI exhibit $\mathrm{V}(\mathrm{GSR}) \leq 0 \mathrm{~km} / \mathrm{s}$, thus corresponding to the $\mathrm{L}$ component.

Neither the $\mathrm{HI}$ nor the $\mathrm{CO}$ observations can provide distances. To estimate distances to the various gas components the only possible way is to find associated objects for which the distance can be determined, i.e. young stars. Association can only be based upon radial velocities. MML studied the association of all young stars and HII regions with well-determined velocities in the exhaustive catalogues of Maurice et al. $(1987,1989)$ with the HI components described above. They consider that objects in the direction of any of the HI components are associated with them if their radial velocity differs from the velocity of the component ridge line at their position by less than $13 \mathrm{~km} / \mathrm{s}$ for FGKM supergiants and HII regions, and by less than $18 \mathrm{~km} / \mathrm{s}$ for OBA stars which have less accurately determined radial velocities. In this way most of the objects can be associated with one component, but a sizeable fraction are not, showing that the gas from which they have been formed has disappeared, probably dispersed by stellar winds and supernova 
explosions in the corresponding regions of star formation. It can be also noted that the objects associated with one gas component are not necessarily distributed like the gas in this component, (see Fig. 4 A-D of MML). This is also so in the LMC (Martin et al. 1976; Prévot et al.(1989).

Once this association is made there are several possibilities. Here is a short summary of what has been done by MML.

1. Interstellar absorption lines in stars belonging to one component can tell about which component is in front. This was first done by Fitzpatrick (1985) who showed that the lowervelocity $\mathrm{HI}$ lies in front of the higher-velocity stars. With more data, MML showed that VL is in front of $\mathrm{L}$ and $\mathrm{L}$ is in front of $\mathrm{H}$, although $\mathrm{H}$ may be in front of $\mathrm{L}$ in the east of the bar; (it may be that $\mathrm{L}$ is warped or made of two sub-components at different distances.) $\mathrm{H}$ and $\mathrm{VH}$ appear mixed together. The very high velocity seen only as interstellar absorption is in front of most of the HI.

2. One can calculate the mean apparent magnitude of similar stars belonging to the different components; this should yield relative distances. One must first correct for interstellar absorption: in fact MML found that the $\mathrm{H}$ stars are more reddened than the $\mathrm{L}$ stars by about $\mathrm{E}(\mathrm{B}-\mathrm{V})=0.05$ mag, confirming that $\mathrm{L}$ is in front of $\mathrm{H}$. The results obtained by MML, after this small correction was made, are disappointing: no significant difference in distance is found between $\mathrm{L}, \mathrm{H}$ and the stars not associated with $\mathrm{HI}$; the corresponding distance moduli are within $0.27 \mathrm{mag}$, thus it is unlikely that the central planes of the components of the SMC extend over more than, say, $10 \mathrm{kpc}$ in depth, a figure about equal to the lateral size of the SMC. There is no trace in these data of a larger distance for the stars in the SW of the bar, but the MML study does not extend as far as the Cepheid studies. No conclusion can be reached for the $\mathrm{K} 1$ region as the number of stars is too small for good statistics.

Cepheids are potentially very interesting for a better understanding of the structure in depth of the SMC. Seven of them are within the limits of the MML study and have accurate mean radial velocities. They can all be assigned to a HI component; the result from them is not conclusive. The mean radial velocities obtained by MFV are, unfortunately, not accurate (quoted error $15 \mathrm{~km} / \mathrm{s}$ ) and assignment to $\mathrm{HI}$ velocity groups is possible for only 40 of them. Using individual distances from MFV, MML found mean distances of 58, 64 and $62 \mathrm{kpc}$ for groups VL, L and $\mathrm{H}$ respectively: this again means no significant distance difference between these groups except perhaps for VL. Using distances from WMMM instead, for the Cepheids in common between MFV and WMMM, MML also found no signifiicant distance differences between the groups. To illustrate the problems encountered, Fig. 1 shows, for some of the Cepheids observed by both MFV and WMMM, their membership of the velocity groups. It is clear that one should not take for granted that radial velocity is directly related to distance, as assumed, for example, by Caldwell and Coulson (1986) in building their Fig. 14. There may, however, be some support for this idea in the data of MFV. For example, in their field 1 in the bar SW, they delineate two regions with coherent velocities of Cepheids: for one region the velocities are in the range of the $\mathrm{H}$ component, and the mean Cepheid distance is higher by $5 \mathrm{kpc}$ with respect to the other region, where the Cepheid velocities are in the range of the L component. Unfortunately, neither the mean Cepheid magnitudes nor their radial velocities are very accurate and better determinations need to be obtained from more measurements at different phases before definitive conclusions can be reached.

\section{Conclusions}

In spite of much observational effort we are still not in a position to produce a three-dimensional picture of the SMC. Most of the atomic gas in the main body of the SMC is distributed in two main complexes plus two secondary ones. The main complex with the larger radial velocity is 
behind the other main complex. Their mean separation is not well known but cannot be larger than 5 to $10 \mathrm{kpc}$. There are young stars associated with either of these complexes, although molecular gas has been detected only in the nearer main complex. The association between young stars and gas is rather loose; there is gas without young stars and also young stars without gas. MML showed that in two well-defined groups of young stars their one-dimensional velocity dispersion is $5 \mathrm{~km} / \mathrm{s}$, comparable to that in the LMC and in $\mathrm{h}$ and $\mathrm{x}$ Per in the Milky Way. Future progress is likely to come from high-quality observations of the mean magnitudes and radial velocities of SMC Cepheids. There are interesting indications for a non-zero depth of the SMC along the line of sight, but most of the existing data are not of sufficiently high quality to be fully conclusive. New systematic observations will certainly be rewarding.

\section{References}

Caldwell, J.A.R., Coulson, I.M. (1986), Mon. Not. R. Astron. Soc. 218, 223.

Feast, M. (1990), these proceedings.

Fitzpatrick, E.L. (1985), Astrophys. J. Suppl. Ser. 59, 77.

Laney, C.D., Stobie, R.S. (1986), Mon. Not. R. Astron. Soc. 222, 449.

Martin, N., Prévot, L., Rebeirot, E., Rousseau, J. (1976), Astron. Astrophys. 51, 31.

Martin, N., Maurice, E., Lequeux, J. (1989), Astron. Astrophys. 215, 219. (MML)

Mathewson, D.S., Ford, V.L. (1984), IAU Symp. 108 Structure and Evolution of the Magellanic

Clouds, S. van den Bergh and K.S. de Boer (eds.) (Reidel: Dordrecht) p.125. (MF)

Mathewson, D.S., Ford, V.L., Visvanathan, N. (1986), Astrophys. J. 301, 664. (MFV)

Mathewson, D.S., Ford, V.L., Visvanathan, N. (1988), Astrophys. J. 333, 617. (MFV)

Maurice, E., et al. (1987), Astron. Astrophys. Suppl. Ser. 67, 423.

Maurice, E., Bouchet, P., Martin, N. (1989), Astron. Astrophys. Suppl. Ser. 78, 445.

McGee, R. X., Newton, L.M. (1981), Proc. Astron. Soc. Aust. 4, 189.

Prévot, L., Rousseau, J., Martin, N. (1989), Astron. Astrophys. 225, 303.

Rubio, M., Garay, G., Montani, J., Thaddeus, P. (1990), Astrophys. J. in preparation.

Tully, R.B., Wolff, S.C. (1984), Astrophys. J. 281, 67.

Welch, D.L., McLaren, R.A., Madore, B.F., McAlary, C.W. (1987), Astrophys. J. 321, 162. (WMMM)

Westerlund, B.E. (1990), Astron. Astrophys. Rev. $2,29$. 